\title{
A systematic review of the prevalence of parental concerns measured by the Parents' Evaluation of Developmental Status (PEDS) indicating developmental risk
}

Susan Woolfenden ${ }^{1,4^{*}}$, Valsamma Eapen ${ }^{2}$, Katrina Williams ${ }^{3}$, Andrew Hayen ${ }^{4}$, Nicholas Spencer ${ }^{5}$ and Lynn Kemp ${ }^{4}$

\begin{abstract}
Background: Parental concerns about their children's development can be used as an indicator of developmental risk. We undertook a systematic review of the prevalence of parents' concerns as an indicator of developmental risk, measured by the Parents' Evaluation of Developmental Status (PEDS) and associated risk factors.

Methods: Electronic databases, bibliographies and websites were searched and experts contacted. Studies were screened for eligibility and study characteristics were extracted independently by two authors. A summary estimate for prevalence was derived. Meta-regression examined the impact of study characteristics and quality. Meta-analysis was used to derive pooled estimates of the impact of biological and psychosocial risk factors on the odds of parental concerns indicating high developmental risk.

Results: Thirty seven studies were identified with a total of 210,242 subjects. Overall 13.8\% (95\% Cl $10.9-16.8 \%)$ of parents had concerns indicating their child was at high developmental risk and 19.8\% (95\% Cl 16.7-22.9\%) had concerns indicating their child was at moderate developmental risk. Male gender, low birth weight, poor/fair child health rating, poor maternal mental health, lower socioeconomic status (SES), minority ethnicity, not being read to, a lack of access to health care and not having health insurance were significantly associated with parental concerns indicating a high developmental risk.
\end{abstract}

Conclusions: The prevalence of parental concerns measured with the PEDS indicating developmental risk is substantial. There is increased prevalence associated with biological and psychosocial adversity.

Trial registration: PROSPERO Registration: CRD42012003215.

Keywords: Prevalence, Parental concerns, Parents Evaluation of Developmental Status (PEDS), Risk factors, Developmental risk, Child health

\section{Background}

Children at developmental risk, are those who have significant problems in at least one area of their development (e.g., motor, language, self-help, social, cognitive, behavioural) [1]. They include children who may be at risk of having a developmental disorder, or children who

\footnotetext{
* Correspondence: susan.woolfenden@sesiahs.health.nsw.gov.au

'Department of Community Child Health, Sydney Children's Hospital

Network, High St Randwick NSW 2031, Sydney, Australia

${ }^{4}$ School of Public Health and Community Medicine, University of New South

Wales, Sydney, Australia

Full list of author information is available at the end of the article
}

are functioning on the lower end of normal who may go on to struggle with the literacy, numeracy and socioemotional demands of school and life [1]. Adverse childhood experiences including socioeconomic disadvantage, poor parental mental health, lack of stimulating early childhood experiences, and lack of access to services can contribute to developmental risk [2-6].

In order to develop a comprehensive public health response to optimise early childhood development, it is helpful if we are able to quantify the state of child development from a population perspective. Although not a comprehensive developmental assessment, measuring parental 
concerns about their children's development can be done in a quick, standardised, systematic manner and has been used to estimate level of developmental risk in the general population and to identify high risk subpopulations $[7,8]$. In addition, eliciting and addressing parental concern is a key component in the family centred practice of detecting individual children at developmental risk in well child health care so that they may have timely referral on for assessment and early intervention prior to starting school [9-12]. The Parents' Evaluation of Developmental Status (PEDS) is a 10 - item parent completed standardised questionnaire, which has been used to elicit parental concerns around child development for children aged less than 8 years in populations, communities and clinical samples. The PEDS open ended questions cover expressive and receptive language, fine motor, gross motor, behaviour, socialisation, self care, and learning [13]. An estimate of developmental risk as high, moderate, low or no risk is derived from the parental concerns recorded and a clinical pathway is recommended. The PEDS has a sensitivity of 91-97\% and specificity of 73-86\% in recent validation studies from the USA for the accuracy of parental concerns in detecting children at high and/or moderate developmental risk [14]. The PEDS has been found to be useful in vulnerable disadvantaged populations, high, middle and low income countries, and has been translated in multiple languages $[14,15]$. There is also a modified version of the PEDS, the Survey PEDS which has 12 close-ended questions that does not allow for further discussion of parental concerns and clinical decision making around these. It is less well validated than the clinical form of the PEDS but is used in telephone population surveys [7,14,16-18].

In order to better understand the current worldwide prevalence of parental concern measured by the PEDS that indicate developmental risk and associated risk factors, we undertook a systematic review to synthesize the available international evidence.

\section{Methods}

\section{Search strategy}

A protocol was developed and registered with the University of York Centre for Reviews and Dissemination (PROSPERO) on 6/11/2012 and updated on the 13/02/2014, registration number CRD42012003215 (http://www.crd.york.ac.uk/PROSPERO/index.asp).

A systematic search of the literature was undertaken using the following inclusion criteria: primary observational studies (cohort study, cross-sectional studies) in geographically defined population or a community sample (including samples from primary health care services) of children aged under 8 years using the PEDS [15] with available prevalence data (Additional file 1). Studies using the modified "Survey PEDS" were also included in this review [14]. Electronic databases searched were Web of Science and Google Scholar, PubMed (Nov 2012), EMBASE (Nov 2012), Medline (Nov 2012), Psychinfo (Nov 2012), Global Health (Nov2012) CINAHL (Nov 2012), the Cochrane Library (Nov 2012), LILACS (Nov 2012), ERIC (Nov 2012), and Proquest (Nov 2012). Secondary searches of citations in review articles, requests to experts in the field and additional searches of the USA based PEDStest and RCH PEDS website for key studies were undertaken. Advice from the Cochrane Child Development, Psychosocial and Learning Groups was sought regarding search terms which were specific for early child development, developmental risk and the $P E D S$. There were no language limitations. Studies using specific clinical samples, for example, neonatal intensive care graduates or with participants who had a known developmental disorder were excluded.

The study titles, abstracts and full papers of "potentially relevant articles" were reviewed independently by two authors (SW\&VE). Disagreements about inclusion were resolved through consensus and discussion with a third author (KW). Study characteristics, prevalence, and risk factors, were extracted independently by SW and VE on a data extraction form that was piloted and modified prior to use. Where insufficient data were reported, study authors were contacted. If no reply was forthcoming or full data not made available, data were included in analysis where possible. Methodological quality was assessed independently by SW and VE based on a validity of the study methods (design, sampling frame, sample size, outcome measures, measurement and response rate), interpretation of the results and applicability of the findings [19], a score of 6 or greater was rated by the reviewers as high quality.

\section{Statistical analysis \\ Prevalence}

Estimates of the prevalence of parental concerns on the PEDS indicating moderate or high risk with corresponding 95\% confidence intervals were extracted from each study. If the confidence intervals were not provided, these were calculated using the Agresti and Coull method [20]. For longitudinal studies, cross-sectional estimates of prevalence were used to extract prevalence data at the first time point. We used an exact likelihood approach to obtain pooled estimates of prevalence. We used metaregression, a regression method that allows the examination of study-level factors on prevalence with the following prespecified variables on prevalence: sample type; type of PEDS; study purpose; study quality; study age group, publication type and country income [21].

\section{Risk factor analysis}

We conducted a meta-analysis for risk factors for having parental concerns on the PEDS indicating high versus 
low/no developmental risk. We extracted odds ratios and $95 \%$ confidence intervals from each study. If odds ratio (OR) with a 95\% confidence interval was not provided, we calculated the odds ratio and 95\% confidence interval. We extracted adjusted odds ratios when possible, but we were unable to calculate these for studies in which they were not provided. We obtained pooled estimates of unadjusted odds ratios (uOR) using metaanalysis with random effects. Where studies presented adjusted odds ratios (aOR) for similar child and family variables these were combined in a separate metaanalysis.

\section{Investigation of heterogeneity}

For all meta-analyses and meta-regressions of prevalence, we modelled within-study variability using the binomial distribution [21]. We then examined heterogeneity through meta-regression models, as described in previous systematic reviews of prevalence [22]. We quantified the reduction in the between study variance from the inclusion of the study characteristics compared to the 'base' model (i.e., the model of prevalence without any covariates). This provides an estimate of the proportion of heterogeneity that is explained by that characteristic. For our meta-analysis of risk factors, the degree of heterogeneity was investigated by estimating the $\mathrm{I}^{2}$ statistic (which describes variation in the summary effect due to genuine variation rather than a sampling error as a percentage, a low $\mathrm{I}^{2}$ indicates low heterogeneity and high $\mathrm{I}^{2}$ indicates significant between study variability) and visual inspection of forest plots [22].

\section{Results}

\section{Studies identified}

The search strategy identified 17,272 titles (excluding duplicates). Seventy-eight articles underwent a text screen and 41 of these were excluded (Figure 1) [23].

\section{Included studies}

The prevalence estimates of the 37 included studies are listed in Table 1 [7,13-18,24-56]. Twenty three studies were published in peer review journals, and the remainder were government/university reports, unpublished abstracts available on the PEDStest website, online population survey data and data from the PEDS Standardisation Manual (2013). There was one longitudinal cohort with data available on samples at two time points three years apart $[39,40,57]$. All other studies were cross sectional.

Fifteen studies used the PEDS as a research tool to measure prevalence of developmental risk of which 12 were population surveys in high income countries and three were community samples. The remaining studies used the PEDS as a developmental surveillance tool in primary health care and early childhood education/early primary school settings [14,24-28,31-33,35,38,41-46,51,53,
58,59]. Eight of the studies were conducted in low and middle income countries [24,42,44-46,51,53,59] and two studies were in socioeconomically disadvantaged communities in the USA [33].

Study sample sizes ranged from 20 to 54602 (median = 467). There were 210,242 subjects in total. Ages ranged from less than 1 month to 7 years and 11 months consistent with the age range for administration of the PEDS. Twenty seven of the studies used translated versions of the PEDS for at least part of their sample.

\section{Study quality}

Quality scores varied between studies (Table 2). Only 13 studies met 6 or more criteria and thus were deemed of high quality $[7,14,16,18,29,34,47-50,52,54,56]$. Key areas of potential bias were lack of random selection of the sample (22/37), a biased sampling frame (20/37), less than 300 participants (11/37), less than $70 \%$ response rate and refusers not described (11/37); confidence intervals not given for prevalence results and lack of subgroup analysis (31/37).

\section{Prevalence of developmental risk}

The pooled estimate of the prevalence of parental concern on the PEDS indicating high developmental risk was $13.8 \%$ (95\% CI 10.9-16.8\%), meaning that almost $14 \%$ of parents raised concerns associated with a high risk for developmental problems (Figure 2). The pooled estimate of for moderate developmental risk was $19.8 \%$ (95\% CI 16.7-22.9\%). The pooled estimate for high or moderate developmental risk was $31.5 \%(95 \%$ CI $27.0-36.0 \%)$, meaning that more than $31 \%$ raised concerns associated with either high or moderate risk of developmental problems.

Meta-regression was conducted for study characteristics (Table 3). Peer reviewed publications had a significantly lower level of parental concerns indicating high developmental risk on the PEDS than unpublished sources (abstracts, reports and population survey data available on the internet). This variable contributed to $19 \%$ of the overall variance between studies. For the report of parental concerns on the PEDS indicating moderate developmental risk, studies done in high income countries reported a significantly higher rate than those done in low and middle income countries. This variable contributed to $29 \%$ of the overall variance between studies. All other variability in study characteristics did not have an impact.

\section{Pooled estimates for biological and psychosocial risk factors}

As shown in Table 4, child sociodemographic variables predictive of parental concerns on the PEDS indicating high developmental risk included male gender [14,16,17, $27,28,30,37,40,47-50,52,54]$, age 3 years and above $[14,27,28,47-50]$, low birth weight $[17,37]$, poor/fair 


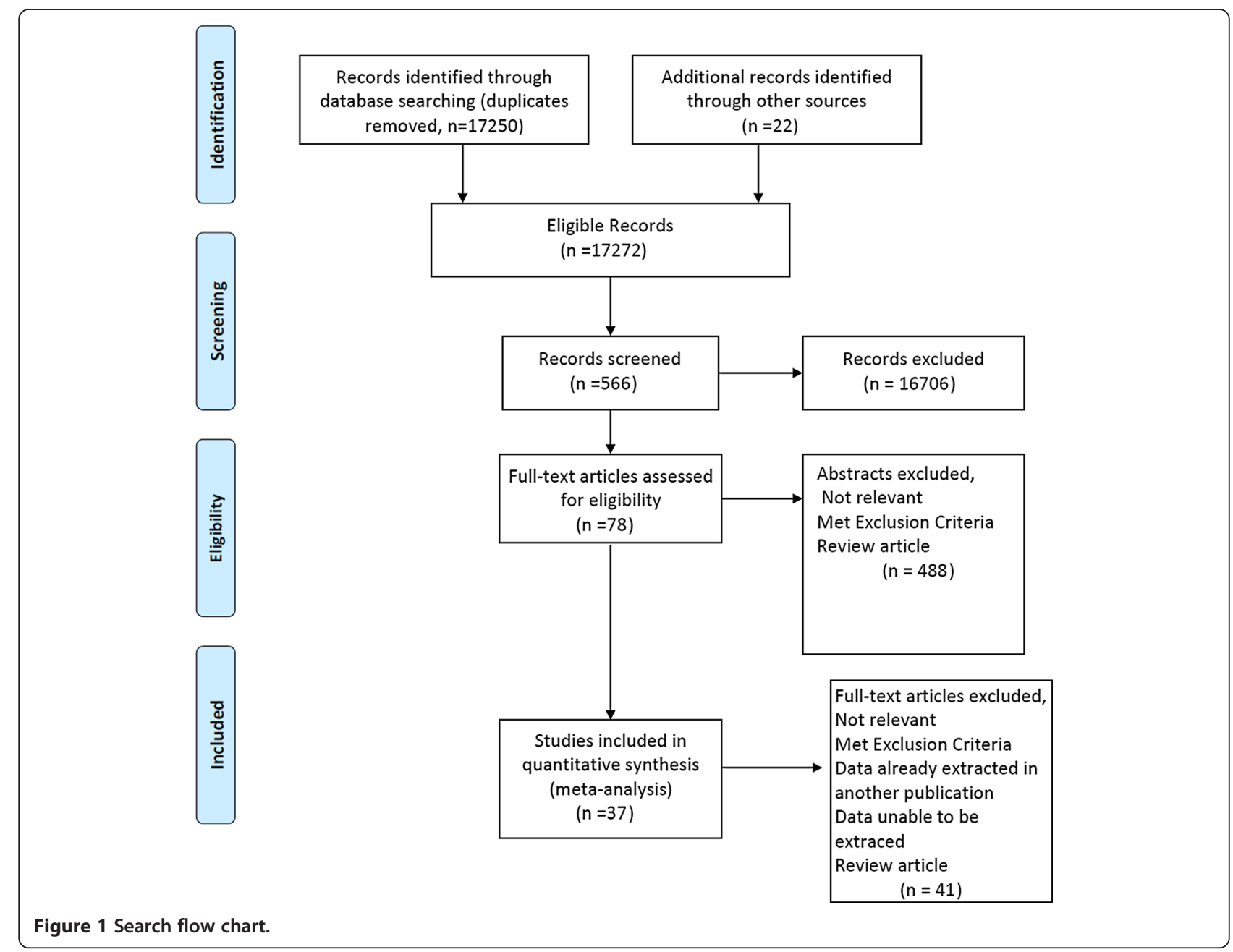

child health [40,47-50] or special health care needs $[16,30]$. Family sociodemographic variables predictive of parental concerns on the PEDS indicating high developmental risk included poor maternal mental health $[7,37,40]$, low family SES [7,16,30,40,47-50], being of African American [7,14,17,30,47-50], Hispanic [7,16,17,30,47-50], First Nations and Australian Aboriginal ethnicity [14,47-50,54], being from a Non English speaking household [30,47-50]. Service level variables predictive of parental concerns on the PEDS indicating high developmental risk included not having a usual source of health care/medical home $[16,30,37,40,47,49,50]$; or having public/no health insurance $[7,16,30,37,47-50]$. Parents not completing high school $[16,27,28,30,40,50]$ and single parenthood [16,40,47-50,54] were significant using unadjusted OR, however not significant as adjusted OR $[17,37]$. Children not being read to daily was significant in the unadjusted analysis [40,47-49], however this did not appear to be significant in the one study that included it in a multivariate analysis $(\mathrm{p}=0.93)$ [40]. Family size (more than 6 people in household) was not significant [47-50]. Parents of children who did not attend formal childcare were less likely to have concerns on the PEDS that indicated high developmental risk [40,47-49], however findings from multivariate analysis of $\mathrm{NSCH} 2007$ data aOR $=1.05$ (CI $0.84,1.33$ ) found a non -significant effect of childcare and that receiving more than 10 hours a week of care at another family's home was a risk factor $(\mathrm{aOR}=1.71, \mathrm{p}<0.05)[17]$.

\section{Narrative summary of single studies, cumulative risk and life course analysis}

A wide range of additional child, family, and service level factors were noted in single studies $[36,37,39,40,56]$. Child level factors were ear infections prior to age 2 $(\mathrm{p}<0.001)$ [40], history of hospital admissions aOR 1.80 (95\% CI 1.35-2.40) [37] and being underweight aOR 2.66 (95\% CI 1.68-4.24) [37]. Family level factors were low scores on contentment/relaxation during pregnancy aOR 2.5 (95\% CI 1.4 -4.2) [39], poor parenting morale when the child was 3 years old aOR 3.9 (95\% CI 2.17.3) [39], maternal history of domestic violence at pregnancy aOR $2.2(95 \%$ CI $1.3-3.7)$ [39,40], household 
Table 1 Included studies characteristics and prevalence rates*

\begin{tabular}{|c|c|c|c|c|c|c|c|c|}
\hline First author & Country & Age (months) & Sample size & Quality score/8 & High risk\% $(95 \% \mathrm{Cl})$ & Moderate risk $\%(95 \% \mathrm{Cl})$ & $\begin{array}{l}\text { High and moderate } \\
\text { risk\% }(95 \% \mathrm{Cl})\end{array}$ & $\begin{array}{l}\text { Low/no } \\
\text { risk\% }(95 \% \mathrm{Cl})\end{array}$ \\
\hline Armstrong [15] & Australia & $0-95$ & 246 & 3 & $11.4(8.0-16.0)$ & $21.9(17.2-27.6)$ & $33.3(27.7-39.5)$ & $66.7(60.5-72.3)$ \\
\hline Bethell [29] & USA & $10-71$ & 22883 & 8 & $9.6(9.2-10.0)$ & $15.9(15.5-16.4)$ & $25.5(25.0-26.1)$ & $74.5(73.9-75.0)$ \\
\hline CHIS [50] & USA & $4-60$ & 2884 & 7 & $25.6(23.6-27.5)$ & $17.4(15.6-19.2$ & $43.0(41.2-44.8)$ & $57.0(55.2-58.8)$ \\
\hline CHIS [49] & USA & $4-60$ & 3029 & 7 & $19.9(18.3-21.5)$ & $18.0(16.4-19.6)$ & $37.9(36.2-39.2)$ & $62.2(60.4-63.9)$ \\
\hline CHIS [48] & USA & $4-60$ & 3058 & 7 & $26.3(24.5-28.2)$ & 18.3(16.7-19.9) & $44.7(72.9-46.4)$ & $55.3(53.6-57.1)$ \\
\hline CHIS [47] & USA & 4 to 60 & 3096 & 7 & $20.1(17.6-22.5)$ & $19.7(17.4-22.0)$ & $39.8(38.1-41.5)$ & $60.2(58.5-61.9)$ \\
\hline Chuan [24] & Malaysia & $12-72$ & 86 & 2 & $26(17.5-35.8)$ & NA & NA & $17.0(10.8-27.0)$ \\
\hline Coghlan [28] & Australia & $18-69$ & 262 & 3 & $9.2(6.2-13.3)$ & $18.7(14.4-23.9)$ & $27.9(22.8-33.6)$ & $72.1(66.4-77.2)$ \\
\hline Davies [25] & UK & $0-24$ & 76 & 5 & $2.6(0.2-9.8)$ & $13.2(7.2-22.8)$ & $15.8(9.2-25.8)$ & $84.2(74.2-90.8)$ \\
\hline Glascoe [32] & USA & $24-84$ & 408 & 5 & NA & NA & $34.6(30.1-39.3)$ & $65.4(60.7-69.9)$ \\
\hline Glascoe [58] & USA & 3-93 (mean 46.5 SD 21.8) & 771 & 5 & $11.0(9-13.4)$ & $26.0(23.0-29.2)$ & $37.0(33.6-40.4)$ & $63.0(59.6-66.4)$ \\
\hline Glascoe [33] & USA & mean 36 & 257 & 4 & $41.0(35.0-47.0)$ & $40.0(34.3-46.2)$ & $81.0(75.6-85.3)$ & $19.0(14.7-24.4)$ \\
\hline Glascoe [33] & USA & mean 36 & 744 & 4 & $23.0(20.1-26.2)$ & $26.0(22.9-29.2)$ & $49.0(45.4-52.5)$ & $51.0(47.5-54.7)$ \\
\hline Glascoe [14] & USA & 0.3-95 (mean 26 SD 20.6) & 47531 & 6 & $4.5(4.3-4.7)$ & $13.7(13.4-14.0)$ & $18.2(17.9-18.6)$ & $81.8(81.5-82.1)$ \\
\hline Gustawan [59] & Indonesia & $3-12$ & 170 & 3 & NA & NA & $31.0(24.2-37.9)$ & $69.0(62.1-75.8)$ \\
\hline Ibironke [56] & USA & 6-71 (mean 38.5 SD 18.4) & 2381 & 7 & NA & NA & $21.4(19.8-23.1)$ & $78.6(76.9-80.2)$ \\
\hline Kiing [41] & Singapore & $1-83$ & 1806 & 3 & $7.5(6.4-8.8)$ & $26.0(24.1-28.1)$ & $33.5(31.4-35.7)$ & $66.0(64.3-68.6)$ \\
\hline Kosht-Fedyshin [42] & Tanzania & $0-60$ & 20 & 4 & $35.0(18.1-56.9)$ & 0.0 & $35.0(18.1-56.9)$ & $65.0(43.1-81.9)$ \\
\hline Limbos [43] & Canada & $12-60$ & 331 & 5 & $13.9(10.6-18.1)$ & $39.6(34.5-45.0)$ & $53.5(48.1-58.8)$ & $46.5(41.2-51.9)$ \\
\hline Malhi [44] & India & $24-60$ & 79 & 2 & NA & NA & $39.2(29.2-50.3)$ & $60.8(49.7-70.8)$ \\
\hline Matibag [45] & Philippines & 24-60 (mean 53.6) & 283 & 2 & $15.0(11.2-19.5)$ & NA & NA & NA \\
\hline McGookin [35] & USA & $9-24$ & 385 & 3 & $5.2(3.4-8.0)$ & $17.4(13.9-21.5)$ & $22.6(18.7-27.1)$ & $77.4(73.0-81.3)$ \\
\hline Ng [18] & Canada & 0-83 (mean 46.1) & 419 & 6 & $9.3(6.9-12.5)$ & $18.9(15.4-22.9)$ & $28.2(24.1-32.7)$ & $72.0(67.3-75.9)$ \\
\hline NSCH (2011/2012) [16] & USA & $4-60$ & 28540 & 8 & $77.0(10.1-11.9)$ & $15.2(14.3-16.1)$ & $26.2(25.7-26.7)$ & $73.8(72.7-75.0)$ \\
\hline Oreto [46] & Philippines & 0-84 (means 53) & 318 & 4 & $15.1(11.6-19.5)$ & $17.0(13.3-21.5)$ & $32.1(27.2-37.4)$ & $67.9(62.6-72.8)$ \\
\hline Palarca [51] & Philippines & 0.5-96 (means 52.6) & 421 & 3 & $9.0(6.6-12.2)$ & $5.0(3.3-7.6)$ & $14.0(11.0-17.7)$ & $86.0(82.3-89.0)$ \\
\hline Restall (2009) [52] & Canada & 60 & 290 & 6 & $13.1(9.7-17.5)$ & $32.4(27.3-38.0)$ & $45.5(39.9-51.3)$ & $54.5(48.7-60.1)$ \\
\hline Rose-Jacobs [37] & USA & $4-36$ & 2010 & 5 & $13.8(12.4-15.4)$ & NA & NA & NA \\
\hline Roux [26] & USA & $<60$ & 2845 & 3 & $28.2(26.6-29.9)$ & $27.5(25.9-29.2)$ & $55.7(53.9-57.5)$ & $44.3(42.5-46.1)$ \\
\hline Sarmiento Campos [31] & Spain & $6-42$ & 1089 & 3 & $8.5(7.0-10.4)$ & $23.0(20.7-25.7)$ & $31.6(28.9-34.4)$ & $68.4(65.6-71.1)$ \\
\hline Sices [38] & USA & 9-31 (means 17.6 SD 6.1) & 60 & 2 & $26.7(17.1-39.1)$ & $10.0(4.4-20.6)$ & $36.7(25.6-49.4)$ & $63.3(50.6-74.4)$ \\
\hline Stevens [7] & USA & 4.35 & 2068 & 6 & $23.4(21.6-25.3)$ & $24.9(23.1-26.8)$ & $48.3(46.2-50.5)$ & $51.7(49.5-53.9)$ \\
\hline
\end{tabular}


Table 1 Included studies characteristics and prevalence rates* (Continued)

\begin{tabular}{|c|c|c|c|c|c|c|c|c|}
\hline Theeranate [53] & Thailand & $0-72$ & 216 & 3 & NA & NA & $4.2(2.1-7.9)$ & $95.8(92.1-97.9)$ \\
\hline Tough [40] & Canada & Mean 38 (SD 8) & 792 & 4 & $10.8(8.9-13.2)$ & $30.2(27.1-33.5)$ & $41.0(37.7-44.5)$ & $59.0(55.5-62.3)$ \\
\hline VSEHQ (2008) [54] & Australia & $60-83$ & 54602 & 6 & $7.2(7.0-7.4)$ & 16.5 (16.2-16.8) & $23.7(23.3-24.0)$ & $76.3(76.0-76.7)$ \\
\hline Wake [55] & Australia & $63.4-90$ & 853 & 3 & NA & NA & $35.0(31.9-38.3)$ & $65.0(61.7-68.1)$ \\
\hline Zuckerman [34] & USA & $<72$ & 24933 & 7 & NA & NA & $22.4(21.9-23.0)$ & $77.6(77.05-78.1)$ \\
\hline
\end{tabular}


Table 2 Quality assessment of included studies*

\begin{tabular}{|c|c|c|c|c|c|c|c|c|c|c|}
\hline $\begin{array}{l}\text { First } \\
\text { author }\end{array}$ & Year & $\begin{array}{l}\text { Random } \\
\text { sample or } \\
\text { whole } \\
\text { population }\end{array}$ & $\begin{array}{l}\text { Unbiased } \\
\text { sampling frame } \\
\text { (i.e. census data) }\end{array}$ & $\begin{array}{l}\text { Adequate } \\
\text { sample size } \\
\text { (>300 subjects) }\end{array}$ & $\begin{array}{l}\text { Measures } \\
\text { were the } \\
\text { standard }\end{array}$ & $\begin{array}{l}\text { Outcomes } \\
\text { measured } \\
\text { by unbiased } \\
\text { assessors }\end{array}$ & $\begin{array}{l}\text { Adequate } \\
\text { response rate } \\
(70 \%) \text { and } \\
\text { refusers } \\
\text { described }\end{array}$ & $\begin{array}{l}\text { Confidence } \\
\text { intervals and } \\
\text { subgroup } \\
\text { analysis }\end{array}$ & $\begin{array}{l}\text { Study } \\
\text { subjects } \\
\text { descirbed }\end{array}$ & $\begin{array}{l}\text { Quality } \\
\text { risk } \\
\text { rating/8 }\end{array}$ \\
\hline Bethell & 2011 & 1 & 1 & 1 & 1 & 1 & 1 & 1 & 1 & 8 \\
\hline $\mathrm{NSCH}$ & $\begin{array}{l}2011 / \\
2012\end{array}$ & 1 & 1 & 1 & 1 & 1 & 1 & 1 & 1 & 8 \\
\hline CHIS & 2003 & 1 & 1 & 1 & 1 & 1 & 1 & 0 & 1 & 7 \\
\hline CHIS & 2005 & 1 & 1 & 1 & 1 & 1 & 1 & 0 & 1 & 7 \\
\hline CHIS & 2009 & 1 & 1 & 1 & 1 & 1 & 1 & 0 & 1 & 7 \\
\hline CHIS & 2007 & 1 & 1 & 1 & 1 & 1 & 1 & 0 & 1 & 7 \\
\hline Ibironke & 2011 & 1 & 1 & 1 & 1 & 1 & 0 & 1 & 1 & 7 \\
\hline Zuckerman & 2009 & 1 & 1 & 1 & 1 & 1 & 0 & 1 & 1 & 7 \\
\hline Glascoe & 2013 & 1 & 1 & 1 & 1 & 1 & 0 & 0 & 1 & 6 \\
\hline Stevens & 2006 & 1 & 1 & 1 & 0 & 1 & 0 & 1 & 1 & 6 \\
\hline $\mathrm{Ng}$ & 2010 & 1 & 1 & 1 & 1 & 1 & 0 & 0 & 1 & 6 \\
\hline Restall & 2009 & 1 & 1 & 0 & 1 & 1 & 0 & 1 & 1 & 6 \\
\hline VSEHQ & 2008 & 1 & 1 & 1 & 1 & 1 & 0 & 0 & 1 & 6 \\
\hline Davies & 2009 & 1 & 1 & 0 & 1 & 1 & 1 & 0 & 0 & 5 \\
\hline Glascoe & 1999 & 0 & 1 & 1 & 1 & 1 & 0 & 0 & 1 & 5 \\
\hline Glascoe & 1997 & 0 & 1 & 1 & 1 & 1 & 0 & 0 & 1 & 5 \\
\hline Rose Jacobs & 2008 & 0 & 0 & 1 & 1 & 1 & 1 & 0 & 1 & 5 \\
\hline Glascoe & 2010 & 0 & 0 & 1 & 1 & 1 & 0 & 0 & 1 & 4 \\
\hline Glascoe & 2010 & 0 & 0 & 1 & 1 & 1 & 0 & 0 & 1 & 4 \\
\hline $\begin{array}{l}\text { Kosht- } \\
\text { Fedyshin }\end{array}$ & 2006 & 1 & 0 & 0 & 1 & 1 & 0 & 0 & 1 & 4 \\
\hline Limbos & 2011 & 0 & 0 & 1 & 1 & 1 & 1 & 0 & 1 & 5 \\
\hline Oreto & 2010 & 0 & 0 & 1 & 1 & 1 & 0 & 0 & 1 & 4 \\
\hline Tough & 2008 & 0 & 0 & 1 & 1 & 1 & 0 & 0 & 1 & 4 \\
\hline Armstrong & 2008 & 0 & 1 & 0 & 1 & & 0 & 0 & 1 & 3 \\
\hline Campos & 2010 & 0 & 0 & 1 & 1 & 1 & 0 & 0 & 0 & 3 \\
\hline Coghlan & 2003 & 0 & 0 & 0 & 1 & 1 & 0 & 0 & 1 & 3 \\
\hline Kiing & 2012 & 0 & 0 & 1 & 1 & 1 & 0 & 0 & 0 & 3 \\
\hline McGookin & 2011 & 0 & 0 & 1 & 1 & 1 & 0 & 0 & 0 & 3 \\
\hline Palarca & 2008 & 0 & 0 & 1 & 1 & 1 & 0 & 0 & 1 & 3 \\
\hline Roux & 2011 & 0 & 0 & 1 & 1 & 1 & 0 & 0 & 0 & 3 \\
\hline Theeranate & 2005 & 0 & 0 & 0 & 1 & 1 & 0 & 0 & 1 & 3 \\
\hline Wake & 2005 & 0 & 0 & 1 & 1 & 1 & 0 & 0 & 0 & 3 \\
\hline Gustawan & 2010 & 0 & 0 & 0 & 1 & 1 & 0 & 0 & 1 & 3 \\
\hline Chuan & 2012 & 0 & 0 & 0 & 1 & 1 & 0 & 0 & 0 & 2 \\
\hline Mahli & 2002 & 0 & 0 & 0 & 1 & 1 & 0 & 0 & 0 & 2 \\
\hline Matibag & 2008 & 0 & 0 & 0 & 1 & 1 & 0 & 0 & 0 & 2 \\
\hline Sices & 2009 & 0 & 0 & 0 & 1 & 1 & 0 & 0 & 0 & 2 \\
\hline
\end{tabular}

\footnotetext{
*Quality rating system as per quality rating tool developed by Public Health Agency in Canada [19].
} 


Armstrong 2004
Bethell 2011
CHIS 2009
CHIS 2007
CHIS 2005
CHIS 2003
Chuan 2012
Coghlan 2003
Davies 2009
Glascoe 2013
Glascoe 2010
Glascoe 2010
Glascoe 1999
Glascoe 1997 *
Gustawan 2010 *
lbironke 2011 *
Kiing 2012
Kosht -Fedyshin 2006
Limbos 2011
Malhi 2002 *
Matibag 2008
McGookin 2011
Ng 2010
NSCH 2011/2012
Oreto 2010
Palarca 2008
Restall 2009
Rose Jacobs 2008
Roux 2011
Sarmiento Campos 2011
Sices 2009
Stevens 2006
Theeranate 2005 *
Tough 2008
VSEHQ 2008
Wake 2005 *
Zuckerman 2009 *
Pooled prevalence

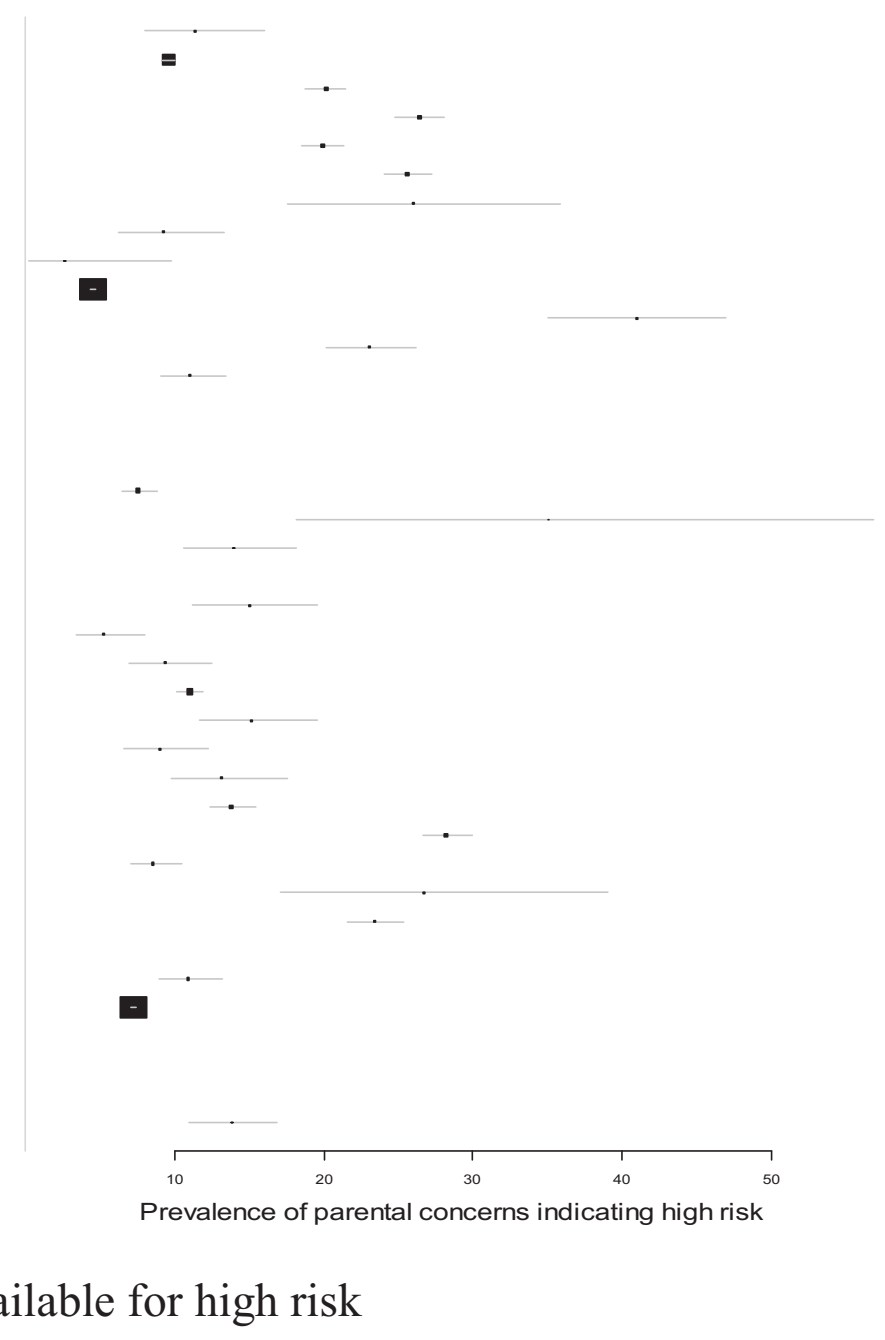

*= no data available for high risk

Figure 2 Prevalence of parental concerns indicating high developmental risk.

food-insecurity (aOR 1.76 (95\% CI 1.26 - 2.46) [37], severe energy insecurity aOR $1.82(95 \% \mathrm{CI} 1.38-2.39)$ [36], geographic site differences in the USA $(\mathrm{p}=0.003)$ [37] and poor overall social support $(p=0.003)$ [39]. Service level factors were referral to early intervention ( $\mathrm{p}<$ $0.001)$, speech pathology ( $\mathrm{p}<0.001)$ or audiology ( $\mathrm{p}<$ 0.001) [40], lack of care coordination aOR 0.33 (95\% CI 0.24-0.46), referrals aOR 0.40 (95\% CI $0.25-0.65)$, family-centred care aOR 0.47 (95\% CI $0.36-0.62$ ) [30] and parental difficulty understanding the doctor uOR 3.35 (95\% CI 2.1-5.4) [48].

Two studies reported a dose-response relationship between the number of risk factors and the increased likelihood of parental concerns on the PEDS indicating high developmental risk $[7,39,40]$. In one study having one risk factor was associated with an aOR 1.7 (95\% CI: 1.20-2.38); two risk factors aOR 3.28, (95\% CI: 2.27-4.73), three risk factors aOR 4.69 (CI: 2.84-7.73), and four risk factors aOR 14.58 (95\% CI: 4.98-42.64) compared to a child with zero risk factors [7]. In addition, the greater the number of risk factors experienced by the child the more likely the child was to not receive comprehensive well child care [7].

The only longitudinal cohort in the review reported that at the second follow up when a child was 5 years of age male gender aOR $2.3(1.3,4.1)$, maternal history of abuse at pregnancy aOR $2.4(1.3,4.4)$ and poor parenting morale when the child was 3 years old aOR3.9 $(2.1,7.3)$ were predictors of parental concerns on the PEDS indicating high developmental risk [39].

\section{Discussion}

Prevalence and associated risk factors for parental concerns on the PEDS indicating developmental risk

This systematic review provides synthesised critically appraised evidence of the substantial global prevalence of parental concerns on the PEDS that indicate high and moderate developmental risk, which increases with biological and psychosocial adversity. This information is 
Table 3 Metaregression of included studies

\begin{tabular}{|c|c|c|c|c|}
\hline Study characteristics & Prevalence of high risk $(\%, 95 \% \mathrm{Cl})$ & $P$ value & Prevalence of moderate risk $(\%, 95 \% \mathrm{Cl})$ & $P$ value \\
\hline All studies & $13.8(10.9,16.8)$ & & $19.9(16.8,23.1)$ & \\
\hline \multicolumn{5}{|l|}{ Sample type } \\
\hline Population survey & $13.5(8.8,18.1)$ & 0.86 & $18.7(14.3,23.1)$ & 0.47 \\
\hline Community sample & $14.0(10.2,17.8)$ & & $21.0(16.7,25.2)$ & \\
\hline \multicolumn{5}{|l|}{ Type of PEDS } \\
\hline Survey PEDS & $17.9(10.9,24.9)$ & 0.15 & $20.6(16.9,24.3)$ & 0.50 \\
\hline Clinical PEDS & $12.6(9.6,15.7)$ & & $18.3(12.9,23.7)$ & \\
\hline \multicolumn{5}{|l|}{ Study purpose } \\
\hline Population risk measure & $14.5(9.7,19.2)$ & 0.71 & $20.1(15.4,24.9)$ & 0.92 \\
\hline Developmental surveillance tool & $13.3(9.6,17.1)$ & & $19.8(15.6,24.0)$ & \\
\hline \multicolumn{5}{|l|}{ Study quality } \\
\hline High quality & $13.5(8.8,18.1)$ & 0.86 & $18.7(14.3,23.1)$ & 0.47 \\
\hline Medium/low quality & $14.0(10.2,17.8)$ & & $21.0(16.7,25.2)$ & \\
\hline \multicolumn{5}{|l|}{ Study age group } \\
\hline 3 years and under & $14.5(8.9,20.2)$ & 0.76 & $22.9(16.7,29.1)$ & 0.25 \\
\hline Includes over 3 years & $13.5(10.1,16.9)$ & & $18.9(15.4,22.3)$ & \\
\hline \multicolumn{5}{|l|}{ Publication type } \\
\hline Peer review/Government report & $11(8.1,14.0)$ & 0.013 & $21.1(16.9,24.4)$ & 0.39 \\
\hline Abstracts/website/manuals & 18.0(13.0,22.9) & & $18.4(14.1,22.8)$ & \\
\hline Country income & High & & & \\
\hline High & $13.2(10.2,16.3)$ & 0.38 & $21.5(18.6,24.5)$ & 0.001 \\
\hline Middle/low & $17.2(8.2,26.1)$ & & $8.4(3.7,13.1)$ & \\
\hline
\end{tabular}

useful for researchers, service providers and clinicians to quantify the level of parental concern and to estimate the risk of children having developmental problems in the general population and to identify vulnerable subpopulations. Gender, low birth weight, poor maternal mental health, low family SES, minority ethnicity, speaking a language other than English and a lack of stimulation, such as a child not being read to, are all associated with adverse impacts on development in the literature and this was supported by the synthesised evidence [1,60-63]. The increasing parental concerns with age of a child regardless of SES demonstrated in this review reflect the increasing developmental demands with age. The impact of child's poor general health on developmental risk may reflect a true increase as some chronic illnesses and syndromes are associated with adverse developmental outcomes. However concerns about their child's health may increase parental concerns generally $[64,65]$.

This review demonstrated that lack of access to usual and comprehensive health care in the USA and Canada was associated with an increased prevalence of parental concerns on the PEDS indicating high developmental risk. Interestingly the evidence for access to services such as early childhood education which has been found to particularly benefit children from disadvantaged backgrounds was not demonstrated [66-68].

Two studies demonstrated that parental concerns on the PEDS indicating high developmental risk increased with the number of risk factors a child was exposed to, consistent with our understanding of the burden of multiple risk factors on early childhood development [7,39,40,62]. In addition, the "inverse care law" applied in one USA study, with the greater the number of risk factors, the less access to comprehensive health care $[7,69]$.

\section{Comparison with other measures of developmental risk}

The confidence intervals around the pooled prevalence estimates of high and moderate developmental risk using the PEDS (27.0-36.0\%) is similar to rates using the Denver Developmental Screening Test (DDST) [70-72] but higher than those using the Australian Early Development Index (AEDI) [1], and Ages and Stages Questionnaire (ASQ) $[38,43,52]$. While the PEDS gives an estimate of high and moderate developmental risk based on parental concerns this is not synonymous with a comprehensive developmental assessment. The PEDS specificities of $73-86 \%$ for parental concerns indicating high and/or moderate developmental risk means that some children identified by parental concern will be false positives $[14,17]$. If parental 
Table 4 Risk factors associated with parental concerns on PEDS indicating high developmental risk

\begin{tabular}{|c|c|c|c|c|}
\hline Risk factor & $\mathrm{N}$ studies & Summary effect OR $(95 \% \mathrm{Cl})$ & $P$ value & Heterogeneity $\left(\mathrm{I}^{2}\right)$ \\
\hline \multicolumn{5}{|l|}{ Child level } \\
\hline Male gender & 11 & $1.68(1.48,1.87)$ & $<0.001$ & $88.1 \%$ \\
\hline Male gender (aOR) & 3 & $2.01(1.38,2.94)$ & $<0.001$ & $91.6 \%$ \\
\hline$>3$ years of age & 7 & $1.83(1.39,2.41)$ & $<0.001$ & $92.7 \%$ \\
\hline Low birth weight & 2 & $1.95(1.53,2.49)$ & $<0.001$ & $0.0 \%$ \\
\hline Poor/fair child health status & 5 & $3.68(2.66,5.08)$ & $<0.001$ & $68 \%$ \\
\hline Special health care needs & 2 & $4.86(2.81,8.38)$ & $<0.001$ & $98 \%$ \\
\hline \multicolumn{5}{|l|}{ Family level } \\
\hline Poor maternal mental health (aOR) & 3 & $1.61(1.31,1.99)$ & $<0.001$ & $0.0 \%$ \\
\hline Low socioeconomic status & 8 & $2.12(1.65,2.72)$ & $<0.001$ & $93.9 \%$ \\
\hline Low socioeconomic status (aOR) & 2 & $1.66(1.08,2.53)$ & 0.019 & $0.0 \%$ \\
\hline Less than high school education & 6 & $1.79(1.14,2.79)$ & 0.011 & $95.5 \%$ \\
\hline Less than high school education (aOR) & 3 & $1.94(0.60,6.23)$ & 0.26 & $98.8 \%$ \\
\hline Being read to less than daily & 4 & $1.47(1.28,1.67)$ & $<0.001$ & $30.6 \%$ \\
\hline Family size ( 6 or more people in household) & 4 & $1.18(0.83,1.68)$ & 0.35 & $91.9 \%$ \\
\hline Single parent & 7 & $1.46(1.16,1.84)$ & 0.001 & $96.8 \%$ \\
\hline Single parent (aOR) & 2 & $0.94(0.75,1.17)$ & 0.57 & $0.0 \%$ \\
\hline \multicolumn{5}{|l|}{ Ethnicity (vs White) } \\
\hline African American & 7 & $1.95(1.43,2.66)$ & $<0.001$ & $90.5 \%$ \\
\hline African American (aOR) & 2 & $1.40(1.10,1.78)$ & 0.006 & $0.0 \%$ \\
\hline Indigenous & 6 & $1.98(1.37,2.86)$ & $<0.001$ & $63.6 \%$ \\
\hline Hispanic & 4 & $2.24(1.83,2.72)$ & $<0.001$ & $88.9 \%$ \\
\hline Hispanic (aOR) & 2 & $1.54(1.16,2.04)$ & 0.03 & $0.0 \%$ \\
\hline \multicolumn{5}{|l|}{ Language spoken } \\
\hline English as a second language (all) & 5 & $1.61(1.12,2.33)$ & 0.01 & $94.2 \%$ \\
\hline English as a second language (Spanish) & 5 & $1.48(0.87,2.53)$ & 0.15 & $96.5 \%$ \\
\hline \multicolumn{5}{|l|}{ Service Level } \\
\hline No Usual Health Care/Medical Home(USA) & 5 & $2.27(1.35,3.81)$ & 0.002 & $84.3 \%$ \\
\hline No or Public Health Insurance & 4 & $1.85(1.45,2.37)$ & $<0.001$ & $95.2 \%$ \\
\hline No Health Insurance (aOR) & 2 & $2.01(1.01,4.02)$ & 0.048 & $57.2 \%$ \\
\hline Does not attend formal childcare & 4 & $0.88(0.77,0.99)$ & 0.040 & $0 \%$ \\
\hline
\end{tabular}

$\mathrm{aOR}=$ adjusted $\mathrm{OR}$.

concerns indicating only high developmental risk are examined the specificity of the PEDS improves to $89 \%$, reducing the number of false positives but the sensitivity drops substantially to less than $50 \%$ giving an unacceptable level of false negatives $[38,43]$. Thus, the true prevalence of actual developmental problems indicated by parental concerns is likely to lie somewhere between the values indicating high and moderate developmental risk $[38,73]$. This is reflected in how the PEDS is used in clinical practice with those children identified as at high developmental risk on parental concerns referred on for a comprehensive developmental assessments and those at moderate risk undergoing a secondary developmental screen with a tool such as the
$A S Q$ and if they fail that then being referred on for a comprehensive developmental assessment [38,43,52]. Systematic reviews of the diagnostic test accuracy (DTA) of the tools that measure developmental risk such as the PEDS in relation to the reference-standard diagnostic batteries in nationally representative samples with an inclusive analysis of vulnerable subpopulations would be useful in understanding how useful developmental risk is as a way to estimate the burden of developmental problems in a population. This systematic review only included studies which had used the PEDS. Prevalence and DTA systematic reviews of other tools such as the $A S Q$ and $A E D I$ would also be useful for further comparison. 


\section{Limitations}

There was considerable variation in quality of the individual studies included in this systematic review. The major sources of bias were an inadequate sampling method, sampling frame, sample size and response rate and a lack of information to aid interpretation and applicability of the results including reporting of confidence intervals and subgroups. It is suggested that future research focus on designing studies that address these issues. Where community samples were used, parents most concerned about their children may be over-represented and this could lead to an overestimation of prevalence. However meta-regression using quality of the study as a variable did not find significant differences in pooled prevalence estimates. There were significant differences in pooled prevalence estimates of developmental risk between studies when the subgroups of publication type and country income were examined. We did not have individual patient data to undertake our own multivariate analysis. Although covariates were similar between studies, how these were measured and the breadth of variables measured varied. This highlights the need for agreed tools on measurement of psychosocial risk in research.

The cross sectional nature of the majority of papers in the review means that only associations of prevalence with risk factors can be examined not causality. In an attempt to address the issue of misclassification bias due to false positives we only examined the relationship between risk factors and high versus low/no developmental risk. Thus any significant relationship is likely to be an underestimate of the true strength of association [17].

\section{Conclusions}

This systematic review found that the prevalence of parental concerns indicating developmental risk on the PEDS is substantial. As with most systematic reviews there were methodological issues with many of the primary studies with variable quality in sampling, representativeness and reporting. Nevertheless, the level of parental concerns that indicate developmental risk highlights the need to support families and promote early childhood development. At the individual level, parents, especially those in disadvantaged communities, should be asked systematically about their concerns and service providers should respond to these concerns through advice, support and facilitating further assessment and early intervention as required $[11,74,75]$. At the population level families should have access to universal high quality early childhood services that optimise child development. Given the prevalence of parental concerns increased with biological and psychosocial adversity, the service response needs to be one of proportionate universalism where the greater the disadvantage, the more services available [68].

\section{Additional file}

Additional file 1: Search strategy.

Abbreviations

PEDS: Parents Evaluation of Developmental Status.

\section{Competing interests}

"Financial Disclosure: The authors have no financial relationships relevant to this article to disclose"

"Potential Non- financial Competing Interests: The authors have no non-financial competing interests relevant to this article to disclose".

\section{Authors' contributions}

SW conceptualized and designed the study, systematically searched the literature, excluded and included papers; designed the data collection form, extracted and analysed the data from the papers, assessed quality of the papers; drafted the initial manuscript and revised all subsequent versions of the manuscript. VE independently excluded and included papers from the systematic literature search assessed their quality and extracted data and reviewed and revised the manuscript. KW provided consensus for SW and VE when difference around exclusion/inclusion of papers and quality scoring and critically reviewed and revised the manuscript. $\mathrm{AH}$ undertook the data analysis of the prevalence data and supported SW in the data analysis of the risk factor data, critically reviewed the manuscript. NS supported SW in the design of the systematic review and critically reviewed and revised the manuscript. LK provided content expertise, supervised SW project management and critically reviewed the manuscript. All authors approved the final manuscript as submitted and agree to be accountable for all aspects of the work.

\section{Acknowledgement}

Many thanks to Professor Frances Glascoe and Associate Professor Sharon Goldfeld for consultation in regards to access to unpublished PEDS data.

\section{What's known on this subject?}

Children at developmental risk are more likely to have long term adverse educational and health outcomes. Parental concerns about their child's development measured by the Parents' Evaluation of Developmental Status (PEDS) has been used as an indicator of developmental risk.

\section{What this study adds}

The prevalence of parental concerns measured with the PEDS that indicate developmental risk is substantial. There is increased prevalence associated with biological and psychosocial adversity.

\section{Author details}

'Department of Community Child Health, Sydney Children's Hospital Network, High St Randwick NSW 2031, Sydney, Australia. ${ }^{2}$ School of Psychiatry, University of New South Wales, Sydney, Australia. ${ }^{3}$ Royal Children's Hospital and Murdoch Children's Research Institute, University of Melbourne, Melbourne, Australia. ${ }^{4}$ School of Public Health and Community Medicine, University of New South Wales, Sydney, Australia. ${ }^{5}$ Warwick Medical School, University of Warwick, Coventry, UK.

Received: 4 April 2014 Accepted: 2 September 2014 Published: 13 September 2014

\section{References}

1. Goldfeld S, O'Connor M, Sayers M, Moore T, Oberklaid F: Prevalence and correlates of special health care needs in a population cohort of australian children at school entry. J Dev Behav Pediatr 2012, 33:319-327.

2. Guralnick MJ: Effectiveness of early intervention for vulnerable children: a developmental perspective. Am J Ment Retard 1997, 102:319-345.

3. King EH, Logsdon DA, Schroeder SR: Risk factors for developmental delay among infants and toddlers. Child Health Care 1992, 21:39-52.

4. Zeanah CH, Boris NW, Larrieu JA: Infant development and developmental risk: a review of the past 10 years. J Am Acad Child Adolesc Psychiatry 1997, 36:165-178. 
5. Patrianakos-Hoobler Al, Msall ME, Marks JD, Huo D, Schreiber MD: Risk factors affecting school readiness in premature infants with respiratory distress syndrome. Pediatrics 2009, 124(1):258-267.

6. Sameroff A: The Transactional Model Of Development: How Children And Contexts Shape Each Other. (pp. 3-21). xiv, 290 pp. In The Transactional Model. Edited by Sameroff AE. Washington, DC, US: American Psychological Association; 2009.

7. Stevens GD: Gradients in the health status and developmental risks of young children: the combined influences of multiple social risk factors. Matern Child Health J 2006, 10:187-199.

8. Zuckerman B, Stevens GD, Inkelas M, Halfon N: Prevalence and correlates of high-quality basic pediatric preventive care. Pediatrics 2004, 114:1522-1529.

9. NH\&MRC: Centre for Community Child Health $(\mathrm{RCH})$ : Child Health Screening and Surveillance: A Critical Review of the Literature. In Canberra: National Health and Medical Research Council; 2002. http://www. nhmrc.gov.au/_files_nhmrc/file/publications/synopses/ch42.pdf accessed Jan 2012.

10. AAP: Identifying infants and young children with developmental disorders in the medical home: an algorithm for developmental surveillance and screening. Pediatrics 2006, 118:405-420.

11. Oberklaid F, Baird G, Blair M, Melhuish E, Hall D: Children's health and development: approaches to early identification and intervention. Arch Dis Child 2013, doi:10.1136/archdischild-2013-304091.

12. National Screening Committee: Child health sub-group report on developmental and behavioural problems. www.screening.nhs.uk/ policydb_download.php?doc=144.

13. Glascoe FP: Using parents' concerns to detect and address developmental and behavioral problems. J Soc Pediatr Nurs 1999, 4:24-35.

14. Glascoe F: Collaborating with Parents: Using Parents' Evaluation of Developmental Status (PEDS) to Detect And Address Developmental And Behavioral Problems. In 2nd edition. Nolensville, TN: PEDSTest.com, LLC; 2013. 2013. (www.pedstest.com).

15. Armstrong M, Goldfeld S: Good Beginnings For Young Children And Families: A Feasibility Study. In City of Wodonga: Centre for Community Child Health (RCH); 2004:1-106.

16. National Survey of Children's Health, Data Resource Center for Child and Adolescent Health, Centre for Disease Control: http://childhealthdata.org/ learn/NSCH accessed Sept2013.

17. Simon AE, Pastor PN, Avila RM, Blumberg SJ: Socioeconomic disadvantage and developmental delay among US children aged 18 months to 5 years. J Epidemiol Community Health 2013, 67:689-695.

18. Ng W, Reynolds D, Kennedy E, Feightner K, Holowaty P, Wade K, Fleiszer P, Northrup D, Glascoe F: Measuring the prevalence of children at risk using; parents' evaluation of developmental status in a telephone survey. Child Indicators Res 2010, 3:167-181.

19. Loney PL, Chambers LW, Bennett KJ, Roberts JG, Stratford PW: Critical appraisal of the health research literature: prevalence or incidence of a health problem. Chronic Dis Can 1998, 19:170-176.

20. Agresti A, Coull B: Approximate is better than 'exact' for interval estimation of binomial proportions. Amer Statist 1998, 52:119-126.

21. Hamza $T$, van Houwelingen $H$, Stijnen $T$ : The binomial distribution of meta-analysis was preferred to model within-study variability. J Clin Epidemiol 2008, 61:41-51.

22. Williams JG, Higgins JP, Brayne CE: Systematic review of prevalence studies of autism spectrum disorders. Arch Dis Child 2006, 91:8-15.

23. Moher D, Liberati A, Tetzlaff J, Altman D, The PRISMA Group: Preferred reporting items for systematic reviews and meta-analyses: the PRISMA statement. PLoS Med 2009, 6:e1000097. doi:1000010.1001371/journal. pmed1000097.

24. Chuan LB, Hock TT, Bin Bujang MA, Haniff J, Chang WS, Abdullah MR, F.P G Parents' Evaluation Of Developmental Status - Validation And Feasbility Of Use Of Its Tranlsated Malay And Mandarin Version. In 2012. http:// www.pedstest.com/Research/tabid/91/articleType/CategoryView/categoryld/ 1/Articles-and-Abstracts.aspx accessed Jan 2013.

25. Davies S, Feeney H: A pilot of the Parents' Evaluation of Developmental Status tool. Community Pract 2009, 82:29-31.

26. Roux A, Herrera P, Wold C, Dunkle M, Glascoe F, Shattuck P: Using 211 to Reach an Underserved Population with Developmental and Autism Screening. Washington University in St. Louis.http://www.pedstest.com/ Research/tabid/91/articleType/CategoryView/categoryld/1/Articles-andAbstracts.aspx accessed Jan 2013. In 2011.
27. Armstrong MF, Goldfeld S: Systems of early detection in Australian communities: the use of a developmental concern questionnaire to link services. Aust J Adv Nurs 2008, 25:36-42.

28. Coghlan D, King J, Wake M: Parents' Evaluation of Developmental Status in the Australian day-care setting:Developmental concerns of parents and carers. J Paediatr Child Health 2003, 39:49-54.

29. Bethell C, Reuland C, Schor E, Abrahms M, Halfon N: Rates of parentcentered developmental screening: disparities and links to services access. Pediatrics 2011, 128:146-155.

30. Coker TR, Shaikh Y, Chung PJ: Parent-reported quality of preventive care for children at-risk for developmental delay. Acad Pediatr 2012, 12:384-390.

31. Sarmiento Campos JA, Squires J, Ponte J: Universal developmental screening: preliminary studies in Galicia, Spain. Early Child Dev Care 2011, 181:475-485.

32. Glascoe FP: The importance of discussing parents' concerns about development: do Parents Express Concerns Spontaneously? Ambul Child Health 1997, 2:349-356.

33. Glascoe FP, Macias M, Herrera P, Brixey S, Simpson P, Li S: How do screening tests perform in settings serving at-risk populations? I Dev Behav Pediatr in press,2010 PEDStest website, http://www.pedstest.com/ Research/tabid/91/articleType/CategoryView/categoryld/1/Articles-andAbstracts.aspx accessed Jan 2013.

34. Zuckerman KE, Boudreau AA, Lipstein EA, Kuhlthau KA, Perrin JM: Household language, parent developmental concerns, and child risk for developmental disorder. Acad Pediatr 2009, 9:97-105.

35. McGookin E, D'Sa V: Developmental screening in a pediatric care practice. Med Health R / 2011, 94:194-196.

36. Cook JT, Frank DA, Casey PH, Rose-Jacobs R, Black MM, Chilton M, DeCuba SE, Appugliese D, Coleman S, Heeren T, Berkowitz C, Cutts DB: A brief indicator of household energy security: associations with food security, child health, and child development in us infants and toddlers. Pediatrics 2008, 122:e867-e875.

37. Rose-Jacobs R, Black MM, Casey PH, Cook JT, Cutts DB, Chilton M, Heeren T, Levenson SM, Meyers AF, Frank DA: Household food insecurity: associations with at-risk infant and toddler development. Pediatrics 2008, 121:65-72.

38. Sices $L$, Stancin T, Kirchner L, Bauchner H: PEDS and ASQ developmental screening tests may not identify the same children. Pediatrics 2009, 124:e640-647

39. Tough SC, Siever JE, Benzies K, Leew S, Johnston DW: Maternal well-being and its association to risk of developmental problems in children at school entry. BMC Pediatr 2010, 10:19. http://www.biomedcentral.com/ content/pdf/1471-2431-10-19.pdf.

40. Tough SC, Siever JE, Leew S, Johnston DW, Benzies K, Clark D: Maternal mental health predicts risk of developmental problems at 3 years of age: follow up of a community based trial. BMC Pregnancy Childbirth 2008, 8(16).

41. Kiing JS, Low PS, Chan YH, Neihart M: Interpreting parents' concerns about their children's development with the Parents Evaluation of Developmental Status: culture matters. J Dev Behav Pediatr 2012, 33:179-183.

42. Kosht-Fedeyshin M: Translation of the Parents' Evaluation of Developmental Status (PEDS) Developmental Screening Tool for Identification of Developmental Delay in Children from Birth to Five Years of Age in the Karagwe District of Northwestern Tanzania, East Africa: a pilot study. Internet J Trop Med 2006, 3. http://ispub.com/IJTM/3/1/10402.

43. Limbos MM, Joyce DP: Comparison of the ASQ and PEDS in screening for developmental delay in children presenting for primary care. J Dev Behav Pediatr 2011, 32:499-511

44. Malhi P, Singh P: Role of Parents' Evaluation of Developmental Status in detecting developmental delay in young children. Indian Pediatr 2002, 39:271-275.

45. Matibag R, Navarro J: Use of Parents Evaluation of Developmental Status (PEDS) Questionnaire Among Pre-School Children aged 2-5 years old: Developmental Concerns of Parents and Teachers. In 2008. http://www. pedstest.com/Research/tabid/91/articleType/CategoryView/categoryld/1/ Articles-and-Abstracts.aspx accessed Jan 2013.

46. Oreto J, Navarro J, Tanchanco L: The use of the Filipino Parents' Evaluation of Developmental Status (PEDS) Among Children aged 0-8 years old. In 2010. http://www.pedstest.com/Research/tabid/91/articleType/ CategoryView/categoryld/1/Articles-and-Abstracts.aspx accessed Jan 2013.

47. California Health Interview Survey. In 2009. http://healthpolicy.ucla.edu/ chis/ accessed Sept 2013 
48. California Health Interview Survey. In 2007. http://healthpolicy.ucla.edu/ chis/ accessed Sept 2013.

49. California Health Interview Survey. In 2005. http://healthpolicy.ucla.edu/ chis/ accessed Sept 2013.

50. California Health Interview Survey. In 2003. http://healthpolicy.ucla.edu/ chis/ accessed Sept 2013

51. Palarca CT: The Role of Parents' Evaluation of Developmental Status (PEDS) As A Screening Tool In The Early Detection Of Developmental Disabilities And Behavioural Disorders In Filipino Children Ages 0-96 Months Old In Gingoong City. In 2008. http://www.pedstest.com/Research/ tabid/91/articleType/CategoryView/categoryld/1/Articles-and-Abstracts.aspx accessed Jan 2013

52. Restall G, Borton B: Parents' concerns about their children's development at school entry. Child Care Health Dev 2010, 36:208-215.

53. Theeranate K, Chuengchitraks S: Parent's Evaluation of Developmental Status (PEDS) detects developmental problems compared to Denver II. J Med Assoc Thai 2005, 88(Suppl 3):S188-192.

54. VSEHQ: Outcomes For Victorian Children At School Entry. State And Local Findings From The School Entrant Health Questionnaire 2008. In Melbourne, Victoria: Data Outcomes and Evaluation Division, Office for Children and Portfolio Coordination, Department of Education and Early Childhood Development; 2011.

55. Wake M, Gerner B, Gallagher S: Does parents' evaluation of developmental status at school entry predict language, achievement, and quality of life 2 years later? Ambul Pediatr 2005, 5:143-149.

56. Ibironke JO, Friedman DS, Repka MX, Katz J, Giordano L, Hawse P, Tielsch JM: Child development and refractive errors in preschool children. Optom Vis Sci 2011, 88:181-187.

57. Tough S, Siever J, Benzies K, Leew S, Johnston DW: Study Report: Screening For Developmental Problems Among Preschool-Aged Children. In Calgary Children's Initiative ; 2006.

58. Glascoe FP: A method for deciding how to respond to parents' concerns about development and behavior... including commentary by Oberklaid F. Ambul Child Health 1999, 5:197-208

59. Gustawan WI, Machfudz S: Validity of parents' evaluation of developmental status (PEDS) in detecting developmental disorders in 3-12 month old infants. Paediatr Indones 2010, 50:6-10.

60. Mistry RS, Benner AD, Biesanz JC, Clark SL, Howes C: Family and social risk, and parental investments during the early childhood years as predictors of low-income children's school readiness outcomes. Early Childhood Res Q 2010, 25:432-449

61. Linver MR, Brooks-Gunn J, Kohen D: Parenting behavior and emotional health as mediators of family poverty effects upon young low-birthweight children's cognitive ability. Ann N Y Acad Sci 1999, 896:376-898.

62. Walker SP, Wachs TD, Grantham-McGregor S, Black MM, Nelson CA, Huffman SL, Baker-Henningham H, Chang SM, Hamadani JD, Lozoff B, Gardner JM, Powell CA, Rahman A, Richter L: Inequality in early childhood: risk and protective factors for early child development. Lancet 2011, 378:1325-1338.

63. Braveman PA, Egerter SA, Woolf SH, Marks JS: When do we know enough to recommend action on the social determinants of health? Am J Prev Med 2011, 40:S58-66.

64. Smart D, Sanson A, Baxter J, Edwards B, Hayes A: Home-To-School Transitions For Financially Disadvantaged Children. In Book Home-ToSchool Transitions For Financially Disadvantaged Children (Editor ed.^eds.). City: The Smith Family; 2008.

65. Glascoe FP: The value of 'parents' evaluations of developmental status' in detecting and addressing children's developmental and behavioral problems. Assess Eff Interv 1998, 23:185-203.

66. Anderson LM, Shinn C, Fullilove MT, Scrimshaw SC, Fielding JE, Normand J, Carande-Kulis VG: The effectiveness of early childhood development programs: a systematic review. Am J Prev Med 2003, 24:32-46.

67. Shonkoff JP: From neurons to neighborhoods: old and new challenges for developmental and behavioral pediatrics. J Dev Behav Pediatr 2003, 24:70-76.

68. Marmot M: Fair Society, Healthy Lives. In The Marmot Review; 2010

69. Hart JT: The inverse care law. Lancet 1971, 1:405-412.

70. Miller JE: Developmental screening scores among preschool-aged children: the roles of poverty and child health. J Urban Health 1998 75(1):135-152.
71. Moraes MW, Weber APR, Santos MCO, Almeida FA: Denver II: evaluation of the development of children treated in the outpatient clinic of Project Einstein in the Community of Paraisópolis. Einstein (Säo Paulo) 2010, 8(2 pt 1):149-153.

72. Nair MK, George B, Padma K, Potti N, Elizabeth KE, Jeyaseelan L: Developmental Evaluation Clinic-CDC experience. Indian Pediatr 2009, 46(Suppl):s63-66.

73. Blanchard LT, Gurka MJ, Blackman JA: Emotional, developmental, and behavioral health of American children and their families: a report from the 2003 National Survey of Children's Health. Pediatrics 2006, 117:e1202-1212

74. Kemp L, Harris E, McMahon C, Matthey S, Vimpani G, Anderson T, Schmied $\checkmark$, Aslam H, Zapart S: Child and family outcomes of a long-term nurse home visitation programme: a randomised controlled trial. Arch Dis Child 2011, 96:533-540.

75. Zoritch B, Roberts I, Oakley A: Day care for pre-school children Cochrane Database Syst Rev 2000, 3:CD000564.

doi:10.1186/1471-2431-14-231

Cite this article as: Woolfenden et al: A systematic review of the prevalence of parental concerns measured by the Parents' Evaluation of Developmental Status (PEDS) indicating developmental risk. BMC Pediatrics 2014 14:231.

\section{Submit your next manuscript to BioMed Central and take full advantage of:}

- Convenient online submission

- Thorough peer review

- No space constraints or color figure charges

- Immediate publication on acceptance

- Inclusion in PubMed, CAS, Scopus and Google Scholar

- Research which is freely available for redistribution 